\title{
A Family of Integer-Point Ternary Parametric Subdivision Schemes
}

\author{
Ghulam Mustafa $\left(\mathbb{D},{ }^{1}\right.$ Muhammad Asghar $\left(\mathbb{D},{ }^{1}\right.$ Shafqat Ali $\left(\mathbb{D},{ }^{1}\right.$ Ayesha Afzal $\left(\mathbb{D},{ }^{2}\right.$ \\ and Jia-Bao Liu (D) $^{3}$ \\ ${ }^{1}$ Department of Mathematics, The Islamia University of Bahawalpur, Bahawalpur, Pakistan \\ ${ }^{2}$ Department of Mathematics, NCBA \& E Sub-Campus Bahawalpur, Bahawalpur, Pakistan \\ ${ }^{3}$ School of Mathematics and Physics, Anhui Jianzhu University, Hefei 230601, China \\ Correspondence should be addressed to Jia-Bao Liu; liujiabaoad@163.com
}

Received 24 April 2021; Revised 6 September 2021; Accepted 28 September 2021; Published 14 October 2021

Academic Editor: M. M. Bhatti

Copyright (c) 2021 Ghulam Mustafa et al. This is an open access article distributed under the Creative Commons Attribution License, which permits unrestricted use, distribution, and reproduction in any medium, provided the original work is properly cited.

\begin{abstract}
New subdivision schemes are always required for the generation of smooth curves and surfaces. The purpose of this paper is to present a general formula for family of parametric ternary subdivision schemes based on the Laurent polynomial method. The different complexity subdivision schemes are obtained by substituting the different values of the parameter. The important properties of the proposed family of subdivision schemes are also presented. The continuity of the proposed family is $C^{2 m}$. Comparison shows that the proposed family of subdivision schemes has higher degree of polynomial generation, degree of polynomial reproduction, and continuity compared with the exiting subdivision schemes. Maple software is used for mathematical calculations and plotting of graphs.
\end{abstract}

\section{Introduction}

Computer aided geometric design (CAGD) is a field which is related to computational mathematics. Subdivision schemes are the important tool of CAGD. Subdivision schemes are used for the generation of smooth curves from initial polygon. In the field of subdivision, the subdivision schemes having three rules are called ternary subdivision schemes. To improve the flexibility of subdivision schemes, some parameters are used in subdivision schemes. Here, we present a brief survey of the parametric ternary subdivision schemes.

In 2002, Hassan et al. [1] purposed a family of interpolating 3-point ternary subdivision schemes with $C^{1}$-continuity. They also compare with binary subdivision schemes. Wang and Qin [2] proposed an improved ternary interpolating subdivision scheme. They also discussed the parameterizations and manipulate the split joint problem with interpolating ternary subdivision scheme. Ko et al. [3] derived a 4-point ternary scheme from cubic polynomial interpolation which has smaller support and higher smoothness, comparing to binary 4-point and 6-point schemes and ternary 3-point and 4-point schemes.

Mustafa and Ashraf [4] presented and analyzed the 6point ternary scheme with the parameter. The proposed scheme has $C^{2}$ continuous over the parametric interval. Mustafa et al. [5] described the general formula for oddpoint parametric ternary subdivision schemes. They proved that the cubic B-spline scheme was a particular case of the suggested ternary scheme. They also demonstrated the effect of the parameter on the limit curve. Pan et al. [6] derived the relation between combined approximating and interpolating subdivision scheme. The resulting curve from the combined subdivision scheme was $C^{2}$ continuous, when analyzed by Laurent polynomial. Siddiqi et al. [7-9] introduced a family of ternary interpolatory and approximating subdivision scheme with one parameter. It was used to generate fractal curves and surfaces. They also presented an algorithm which produced ternary $m$-point approximating subdivision schemes by taking $m$ as integer greater than 2 with the help of uniform $B$-spline blending functions. 
Rehan and Siddiqi [10] presented a family of 3-point and 4-point ternary approximating subdivision schemes. They also analyzed degree of smoothness of a family of ternary 3point and 4-point subdivision schemes which are $C^{1}$ and $C^{3}$ continuity, respectively. Ashraf et al. $[11,12]$ presented and analyzed the geometrical properties of the 4-point ternary interpolating subdivision scheme. This scheme involves a tension parameter. They derived the conditions on the tension parameter and initial control polygon that permitted the creation of positivity and monotonicity-preserving curves after a finite number of subdivision steps.

In 2021, Tan and Tong [13] presented a nonstationary 4point ternary interpolating subdivision scheme. They also discussed the shape preserving properties of the proposed subdivision scheme. Tariq et al. [14] presented a unified class of combined subdivision schemes with two-shape control parameters in order to grow versatility for overseeing valuable necessities. Hameed et al. [15] presented a new method to construct a family of $(2 N+2)$-point binary subdivision schemes with one tension parameter. Mustafa et al. [16] presented a subdivision collocation method to resolve Bratu's boundary value problem by using the approximating subdivision scheme.

1.1. Our Contributions. The main purpose of this work is to present a generalized formula for derivation of parametric ternary subdivision schemes. Our proposed high complexity schemes have high values of continuity, degree of generation, and degree of reproduction of polynomials. The claim is proved in comparison section (see Table 1). Our schemes give better approximation and smoothness when compared with the same type of exiting subdivision schemes (see Figure 1).

The paper is organized as follows. In Section 2, we present the general formula and derivations of family of ternary subdivision schemes. Analysis of the proposed family is presented in Section 3. Section 4 is for comparison of proposed family of subdivision schemes with exiting subdivision schemes. Conclusions are drawn in Sections 5.

\section{A Family of Integer-Point Ternary Schemes}

In this section, we propose a general formula for integerpoint ternary approximating subdivision schemes with one parameter in the form of Laurent polynomial:

$$
\begin{aligned}
A_{m}(z)= & \frac{1}{3^{2 m-1}}\left(1+z+z^{2}\right)^{2 m+1} \\
& \cdot\left[\frac{1}{12}+w+\left(\frac{1}{6}-2 w\right) z+\left(\frac{1}{12}+w\right) z^{2}\right],
\end{aligned}
$$

where $m=0,1,2$, and 3. By putting $m=0$ in (1), we get the Laurent polynomial of a 2-point interpolating subdivision scheme:

$$
A_{0}(z)=\left[\frac{1}{4}+3 w+\left(\frac{3}{4}-3 w\right) z+z^{2}+\left(\frac{3}{4}-3 w\right) z^{3}+\left(\frac{1}{4}+3 w\right) z^{4}\right] .
$$

The scheme corresponding to the Laurent polynomial (2) is

$$
\begin{aligned}
f_{3 i}^{k+1} & =f_{i}^{k}, \\
f_{3 i+1}^{k+1} & =\left(\frac{3}{4}-3 w\right) f_{i}^{k}+\left(\frac{1}{4}+3 w\right) f_{i+1}^{k}, \\
f_{3 i+2}^{k+1} & =\left(\frac{1}{4}+3 w\right) f_{i}^{k}+\left(\frac{3}{4}-3 w\right) f_{i+1}^{k} .
\end{aligned}
$$

By putting $m=1$ in (1), we get the Laurent polynomial of a 3-point approximating subdivision scheme:

$$
\begin{aligned}
A_{1}(z)= & {\left[\left(\frac{1}{36}+\frac{1}{3} w\right)+\left(\frac{5}{36}+\frac{1}{3} w\right) z+\left(\frac{13}{36}+\frac{1}{3} w\right) z^{2}\right.} \\
& +\left(\frac{11}{18}-\frac{2}{3} w\right) z^{3}+\left(\frac{13}{18}-\frac{2}{3} w\right) z^{4}+\left(\frac{11}{18}-\frac{2}{3} w\right) z^{5} \\
& \left.+\left(\frac{13}{36}+\frac{1}{3} w\right) z^{6}+\left(\frac{5}{36}+\frac{1}{3} w\right) z^{7}+\left(\frac{1}{36}+\frac{1}{3} w\right) z^{8}\right]
\end{aligned}
$$

(4) is

The scheme corresponding to the Laurent polynomial

$$
\begin{aligned}
& f_{3 i}^{k+1}=\left(\frac{13}{36}+\frac{1}{3} w\right) f_{i-1}^{k}+\left(\frac{11}{18}-\frac{2}{3} w\right) f_{i}^{k}+\left(\frac{1}{36}+\frac{1}{3} w\right) f_{i+1}^{k} \\
& f_{3 i+1}^{k+1}=\left(\frac{5}{36}+\frac{1}{3} w\right) f_{i-1}^{k}+\left(\frac{13}{18}-\frac{2}{3} w\right) f_{i}^{k}+\left(\frac{5}{36}+\frac{1}{3} w\right) f_{i+1}^{k} \\
& f_{3 i+2}^{k+1}=\left(\frac{1}{36}+\frac{1}{3} w\right) f_{i-1}^{k}+\left(\frac{11}{18}-\frac{2}{3} w\right) f_{i}^{k}+\left(\frac{13}{36}+\frac{1}{3} w\right) f_{i+1}^{k}
\end{aligned}
$$

By putting $m=2$ in (1), we get the Laurent polynomial of a 5-point approximating subdivision scheme:

$$
\begin{aligned}
A_{2}(z)= & {\left[\left(\frac{1}{324}+\frac{1}{27} w\right)+\left(\frac{7}{324}+\frac{1}{9} w\right) z+\left(\frac{13}{162}+\frac{2}{9} w\right) z^{2}\right.} \\
& +\left(\frac{65}{324}+\frac{5}{27} w\right) z^{3}+\frac{10}{27} z^{4}+\left(\frac{19}{36}-\frac{1}{3} w\right) z^{5} \\
& +\left(\frac{16}{27}-\frac{4}{9} w\right) z^{6} \\
& +\left(\frac{19}{36}-\frac{1}{3} w\right) z^{7}+\frac{10}{27} z^{8}+\left(\frac{65}{324}+\frac{5}{27} w\right) z^{9} \\
& +\left(\frac{13}{162}+\frac{2}{9} w\right) z^{10} \\
& \left.+\left(\frac{7}{324}+\frac{1}{9} w\right) z^{11}+\left(\frac{1}{324}+\frac{1}{27} w\right) z^{12}\right] .
\end{aligned}
$$

(6) is

The scheme corresponding to the Laurent polynomial 
TABLE 1: The comparison of a family of ternary schemes $S_{m}$, where $C_{s}, C$, and $G_{d}$ are complexity, continuity analysis, and generation degree of subdivision schemes, respectively.

\begin{tabular}{|c|c|c|c|c|}
\hline$C_{s}$ & Schemes & Type & $C$ & $G_{d}$ \\
\hline 3-point & $A_{1}$ scheme & Approx & $C^{2}$ for $w \in(-(3 / 36),(9 / 36))$ & Quadratic \\
\hline 3-point & Scheme [17] & Approx & $C^{1}$ for $w \in(0,(1 / 2))$ & Linear \\
\hline 3-point & Scheme [1] & Int & $C^{1}$ & Linear \\
\hline 3-point & Scheme [18] & Approx & $C^{2}$ & Quadratic \\
\hline 3-point & Scheme $[10]$ & Approx & $C^{1}$ & Linear \\
\hline 3-point & Scheme [5] & Approx & $C^{2}$ for $w \in(-(1 / 12),(11 / 2))$ & - \\
\hline 3-point & Scheme [9] & Approx & $C^{2}$ & - \\
\hline 5-point & $A_{2}$ scheme & Approx & $C^{4}$ for $w \in(-(3 / 36),(9 / 36))$ & Quartic \\
\hline 5 -point & Scheme [17] & Approx & $C^{4}$ for $w \in(0,(1 / 2))$ & Quartic \\
\hline 5-point & Scheme $[18]$ & Int & $C^{1}$ & Linear \\
\hline 5 -point & Scheme $[5]$ & Approx & $C^{3}$ for $\mu \in((53 / 8748),(187 / 17496))$ & Cubic \\
\hline 5-point & Scheme [9] & Approx & $C^{4}$ & - \\
\hline 6-point & $A_{3}$ scheme & Approx & $C^{6}$ for $w \in(-(3 / 36),(9 / 36))$ & Sextic \\
\hline 6-point & Scheme [4] & Int & $C^{2}$ & Linear \\
\hline 6-point & Scheme [9] & Approx & $C^{5}$ & Quadratic \\
\hline
\end{tabular}
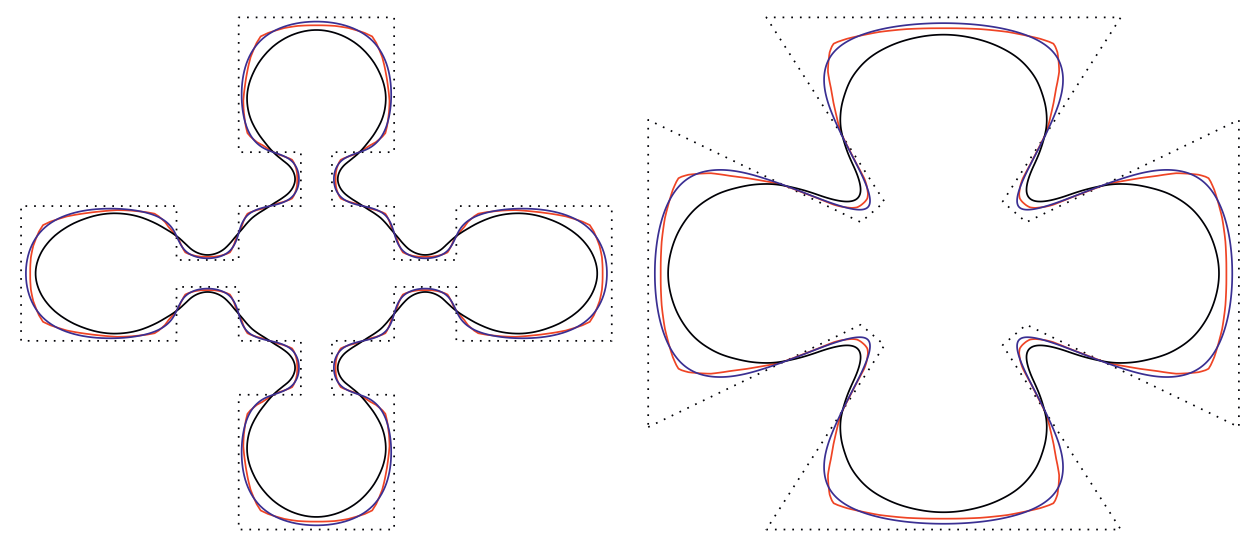

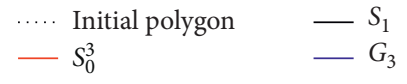

(a)
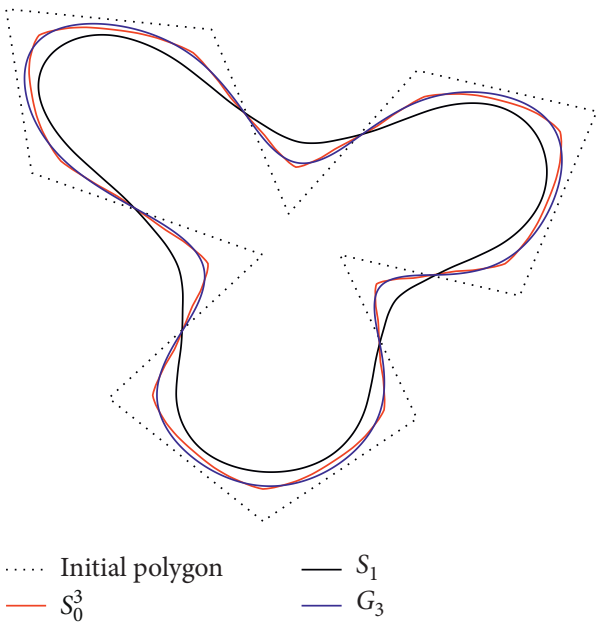

(c)

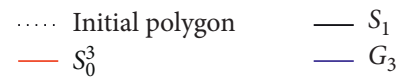

(b)

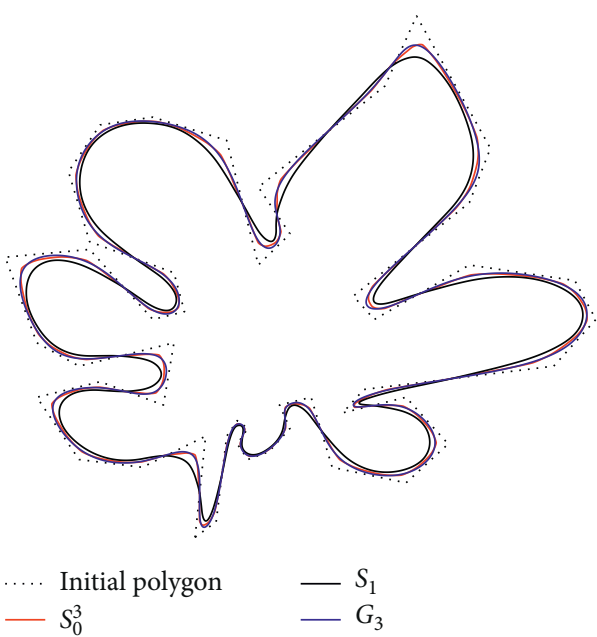

(d)

Figure 1: (a-d) Comparison of limit curves for close polygons produced by 3-point schemes $S_{0}^{3}, S_{1}$, and $G_{3}$. 


$$
\begin{aligned}
f_{3 i}^{k+1}= & \left(\frac{13}{162}+\frac{2}{9} w\right) f_{i-2}^{k}+\left(\frac{19}{36}-\frac{1}{3} w\right) f_{i-1}^{k}+\frac{10}{27} f_{i}^{k} \\
& +\left(\frac{7}{324}+\frac{1}{9} w\right) f_{i+1}^{k}, \\
f_{3 i+1}^{k+1}= & \left(\frac{7}{324}+\frac{1}{9} w\right) f_{i-2}^{k}+\frac{10}{27} f_{i-1}^{k}+\left(\frac{19}{36}-\frac{1}{3} w\right) f_{i}^{k} \\
& +\left(\frac{13}{162}+\frac{2}{9} w\right) f_{i+1}^{k}, \\
f_{3 i+2}^{k+1}= & \left(\frac{1}{324}+\frac{1}{27} w\right) f_{i-2}^{k}+\left(\frac{65}{324}+\frac{5}{27} w\right) f_{i-1}^{k} \\
& +\left(\frac{16}{27}-\frac{4}{9} w\right) f_{i}^{k} \\
& +\left(\frac{65}{324}+\frac{5}{27} w\right) f_{i+1}^{k}+\left(\frac{1}{324}+\frac{1}{27} w\right) f_{i+2}^{k} .
\end{aligned}
$$

By putting $m=3$ in (1), we get the Laurent polynomial of a 6-point approximating subdivision scheme:

$$
\begin{aligned}
A_{3}(z)= & \left(\frac{1}{2916}+\frac{1}{243} w\right)+\left(\frac{1}{324}+\frac{5}{243} w\right) z \\
& +\left(\frac{43}{2916}+\frac{5}{81} w\right) z^{2} \\
& +\left(\frac{35}{729}+\frac{28}{243} w\right) z^{3}+\left(\frac{343}{2916}+\frac{35}{243} w\right) z^{4} \\
& +\left(\frac{665}{2916}+\frac{7}{81} w\right) z^{5} \\
& +\left(\frac{175}{486}-\frac{14}{243} w\right) z^{6}+\left(\frac{1373}{2916}-\frac{55}{243} w\right) z^{7} \\
& +\left(\frac{125}{243}-\frac{8}{27} w\right) z^{8} \\
& +\left(\frac{1373}{2916}-\frac{55}{243} w\right) z^{9}+\left(\frac{175}{486}-\frac{14}{243} w\right) z^{10} \\
& +\left(\frac{665}{2916}+\frac{7}{81} w\right) z^{11} \\
& +\left(\frac{343}{2916}+\frac{35}{243} w\right) z^{12}+\left(\frac{35}{729}+\frac{28}{243} w\right) z^{13} \\
& +z^{14} \\
& \left.\left.+\left(\frac{1}{291} w\right) z^{15}+\frac{1}{243} w\right) z^{16}\right]
\end{aligned}
$$

(8) is

$$
\begin{aligned}
& f_{3 i}^{k+1}=\left(\frac{43}{2916}+\frac{5}{81} w\right) f_{i-2}^{k}+\left(\frac{665}{2916}+\frac{7}{81} w\right) f_{i-1}^{k} \\
& +\left(\frac{125}{243}-\frac{8}{27} w\right) f_{i}^{k} \\
& +\left(\frac{665}{2916}+\frac{7}{81} w\right) f_{i+1}^{k}+\left(\frac{43}{2916}+\frac{5}{81} w\right) f_{i+2}^{k}, \\
& f_{3 i+1}^{k+1}=\left(\frac{1}{324}+\frac{5}{243} w\right) f_{i-2}^{k}+\left(\frac{343}{2916}+\frac{35}{243} w\right) f_{i-1}^{k} \\
& +\left(\frac{1373}{2916}-\frac{55}{243} w\right) f_{i}^{k} \\
& +\left(\frac{175}{486}-\frac{14}{243} w\right) f_{i+1}^{k}+\left(\frac{35}{729}+\frac{28}{243} w\right) f_{i+2}^{k} \\
& +\left(\frac{1}{2916}+\frac{1}{243} w\right) f_{i+3}^{k} \\
& f_{3 i+2}^{k+1}=\left(\frac{1}{2916}+\frac{1}{243} w\right) f_{i-2}^{k}+\left(\frac{35}{729}+\frac{28}{243} w\right) f_{i-1}^{k} \\
& +\left(\frac{175}{486}-\frac{14}{243} w\right) f_{i}^{k} \\
& +\left(\frac{1373}{2916}-\frac{55}{243} w\right) f_{i+1}^{k}+\left(\frac{343}{2916}+\frac{35}{243} w\right) f_{i+2}^{k} \\
& +\left(\frac{1}{324}+\frac{5}{243} w\right) f_{i+3}^{k} \text {. }
\end{aligned}
$$

Similarly by substituting the different values of $m$, we get the family members of ternary subdivision schemes. Table 2 contains the mask of proposed family members of ternary subdivision schemes corresponding to $m=0,1,2$, and 3 .

\section{Analysis of Integer-Point Ternary Schemes}

This section contains analysis of important properties of proposed subdivision schemes. Laurent polynomial [19] is used to compute the degree of generation, degree of reproduction, and continuity analysis. While Rioul's method [20] is used to compute lower and upper bounds on Hölder regularity of the scheme corresponding to $A_{m}(z)$.

Theorem 1. Whenever convergent and generating cubic polynomials, the family of ternary subdivision schemes (1) has cubic degree of reproduction with respect to the primal parametrization.

Proof. By taking the derivative of (1) with respect to $z$, we obtain 
TABLE 2: The mask of integer-point ternary subdivision schemes $A_{m}$ corresponding to the different values of $m$.

\begin{tabular}{|c|c|}
\hline$m$ & Mask \\
\hline 0 & $A_{0}=\{((1 / 4)+3 w),((3 / 4)-3 w), 1,((3 / 4)-3 w),((1 / 4)+3 w)\}$ \\
\hline 1 & $\begin{array}{c}A_{1}=\{((1 / 36)+(1 / 3) w),((5 / 36)+(1 / 3) w),((13 / 36)+(1 / 3) w),((11 / 18)-(2 / 3) w),((13 / 18)-(2 / 3) w),((11 / 18)-(2 / 3) w) \\
((13 / 36)+(1 / 3) w),((5 / 36)+(1 / 3) w),((1 / 36)+(1 / 3) w)\}\end{array}$ \\
\hline 2 & $\begin{array}{c}A_{2}=\{((1 / 324)+(1 / 27) w),((7 / 324)+(1 / 9) w),((13 / 162)+(2 / 9) w),((65 / 324)+(5 / 27) w),(10 / 27),((19 / 36)-(1 / 3) w) \\
\quad((16 / 27)-(4 / 9) w),((19 / 36)-(1 / 3) w),(10 / 27),((65 / 324)+(5 / 27) w),((13 / 162)+(2 / 9) w),((7 / 324)+(1 / 9) w) \\
((1 / 324)+(1 / 27) w)\}\end{array}$ \\
\hline 3 & $\begin{array}{c}A_{3}=\{((1 / 2916)+(1 / 243) w),((1 / 324)+(5 / 243) w),((43 / 2916)+(5 / 81) w),((35 / 729)+(28 / 243) w),((343 / 2916)+(35 / 243) w) \\
((665 / 2916)+(7 / 81) w),((175 / 486)-(14 / 243) w),((1373 / 2916)-(55 / 243) w),((125 / 243)-(8 / 27) w),((1373 / 2916)-(55 / 243) w) \\
((175 / 486)-(14 / 243) w),((665 / 2916)+(7 / 81) w),((343 / 2916)+(35 / 243) w),((35 / 729)+(28 / 243) w),((43 / 2916)+(5 / 81) w) \\
((1 / 324)+(5 / 243) w),((1 / 2916)+(1 / 243) w)\}\end{array}$ \\
\hline
\end{tabular}

TABLE 3: The degree of generation and degree of reproduction w.r.t parameterization for different values of $m$.

\begin{tabular}{ccccc}
\hline$m$ & $G_{d}$ & $\tau$ & $R_{d}$ & Parameterization \\
\hline 1 & 2 & 4 & Quadratic, when $w=-(5 / 12)$ & Primal \\
2 & 4 & 6 & Cubic, when $w=-(23 / 36)$ & Primal \\
3 & 6 & 8 & Cubic, when $w=-(31 / 36)$ & Primal \\
\hline
\end{tabular}

$$
\begin{aligned}
A_{m}^{\prime}(z)= & \frac{1}{3^{2 m-1}\left(1+z+z^{2}\right)}\left(\left(1+z+z^{2}\right)^{2 m+1}(2 m+1)(1+2 z)\left(\frac{1}{12}+w\right)\right. \\
& \left.\left.+\left(\frac{1}{6}-2 w\right) z+\left(\frac{1}{12}+w\right) z^{2}\right)\right)+\frac{1}{3^{2 m-1}}\left(1+z+z^{2}\right)^{2 m+1}\left(\frac{1}{6}-2 w\right. \\
& \left.+\left(\frac{1}{6}+w\right) z\right) .
\end{aligned}
$$

After substituting $z=1$ in (4) and (10, we get $A_{m}(1)=3$ and $A_{m}^{\prime}(1)=6 m+6$. After taking the first three derivatives of (10) and substituting $z=1$, we have

$$
\begin{aligned}
& A_{m}^{\prime \prime}(1)=12 m^{2}+22 m+\frac{19}{2}+18 w, \\
& A_{m}^{\prime \prime}(1)=24 m^{3}+60 m^{2}+45 m+\frac{21}{2}+108 m w+54 w .
\end{aligned}
$$

The value of shift parameter $\tau=\left(A_{m}^{\prime}(1) / 3\right)=2 m+2$. Hence, by [19], the subdivision schemes corresponding to (1) have primal parametrization. Furthermore, for different values of $m$, we can easily verify that

$$
A_{m}^{k}(1)=3 \prod_{j=0}^{k-1}(2 m+2-j) \quad \text { for all } k=1,2,3 .
$$

Hence, by [19], the schemes corresponding to $A_{m}(z)$ have cubic degree of reproduction with respect to the primal parametrization.

Table 3 summarizes the results of degree of generations, shift parameter, degree of reproductions, and parametrization of proposed family of integer-point ternary subdivision schemes. Here, $m, G_{d}, \tau$, and $R_{d}$ and parametrization denote the positive integer, degree of generation, shift parameter, degree of reproduction, and parametrization of the scheme, respectively.

Theorem 2. The family of ternary approximating subdivision schemes (1) has $C^{2 m}$ continuity when $w \in(-(3 / 36),(9 / 36))$.

Proof. For $C^{2 m}$ continuity of the schemes corresponding to $A_{m}(z)$, consider the Laurent polynomial:

$$
A_{m}^{1}(z)=\left(\frac{3 z^{2}}{1+z+z^{2}}\right)^{2 m+1} A_{m}(z)
$$

where $A_{m}(z)$ is defined in (1). This implies

$$
\begin{aligned}
A_{m}^{1}(z)= & \left(\frac{3 z^{2}}{1+z+z^{2}}\right)^{2 m+1} \frac{1}{3^{2 m-1}}\left(1+z+z^{2}\right)^{2 m+1} \\
& \cdot\left[\frac{1}{12}+w+\left(\frac{1}{6}-2 w\right) z\right. \\
& \left.+\left(\frac{1}{12}+w\right) z^{2}\right]
\end{aligned}
$$

After simplification, we obtain 


$$
A_{m}^{1}(z)=9 z^{4 m+2}\left[\frac{1}{12}+w+\left(\frac{1}{6}-2 w\right) z+\left(\frac{1}{12}+w\right) z^{2}\right]
$$

Let $A_{m}^{1}$ be the mask of the scheme $S_{m}$ corresponding to $A_{m}^{1}(z)$; then, we have

$$
A_{m}^{1}=\left[\frac{9}{12}+9 w, \frac{9}{6}-18 w, \frac{9}{12}+9 w\right]
$$

The scheme corresponding to $A_{m}^{1}(z)$ is $C^{2 m}$ continuous if $\left\|(1 / 3) S_{m}\right\|_{\infty}<1$, for this, we have to check that

$$
\left\|\frac{1}{3} S_{m}\right\|_{\infty}=\max \left\{\left|\frac{9}{36}+3 w\right|,\left|\frac{9}{18}-6 w\right|,\left|\frac{9}{36}+3 w\right|\right\}<1 .
$$

$\left\|(1 / 3) S_{m}\right\|_{\infty}<1 \quad$ when $\quad w \in(-(3 / 36),(9 / 36))$. The schemes corresponding to $A_{m}(z)$ has $C^{2 m}$ continuity which completes the proof.

Theorem 3. The Hölder regularity of a family of schemes $S_{m}$ corresponding to (1) is $r=2 m+1-\log _{3}(\mu)$, where $\mu$ is defined as

$$
\begin{cases}\mu=\frac{3}{2}-18 w, & \text { if }-\frac{3}{36}<w \leq \frac{1}{36} \\ \mu=\frac{3}{4}+9 w & \text { if } \frac{1}{36}<w<\frac{9}{36}\end{cases}
$$

Proof. The Laurent polynomial (1) can be written as

$$
A_{m}(z)=\left(\frac{1+z+z^{2}}{3}\right)^{2 m+1} b_{m}(z)
$$

where

$$
b_{m}(z)=\left[\frac{3}{4}+9 w+\left(\frac{9}{6}-18 w\right) z+\left(\frac{3}{4}+9 w\right) z^{2}\right] .
$$

From (20), the coefficients of $z$ in $b(z)$ are $b_{0}=(3 / 4)+9 w, b_{1}=(9 / 6)-18 w$, and $b_{2}=(3 / 4)+9 w$. The number of factors in $A_{m}(z)$ are $k=2 m+1$. The matrices $B_{n}$ of order $2 \times 2$, where $n=0,1,2$ and the elements of the matrices $B_{0}, B_{1}$, and $B_{2}$ can be derived by $\left(B_{n}\right)_{i j}=b_{(2+n)+i-3 j}$, for $i, j=1$ and 2 , we have

$$
\begin{aligned}
B_{0} & =\left(\begin{array}{cc}
\frac{3}{4}+9 w & 0 \\
\frac{6}{4}-18 w & 0
\end{array}\right), \\
B_{1} & =\left(\begin{array}{cc}
\frac{6}{4}-18 w & 0 \\
\frac{3}{4}+9 w & 1
\end{array}\right), \\
\& B_{2} & =\left(\begin{array}{cc}
\frac{3}{4}+9 w & 0 \\
0 & \frac{3}{4}+9 w
\end{array}\right) .
\end{aligned}
$$

The eigenvalues of $B_{0}, B_{1}$, and $B_{2}$ are $\{0,(3 / 4)+9 w\},\{0,(3 / 2)-18 w\}$, and $\{(3 / 4)+9 w,(3 / 4)+$ $9 w\}$, respectively. For bounds on Hölder regularity, we calculate $\max \left\{\rho\left(B_{0}\right), \rho\left(B_{1}\right), \rho\left(B_{2}\right)\right\} \leq \mu \leq \max \left\{\left\|\left(B_{0}\right)\right\|\right.$, $\left.\left\|\left(B_{1}\right)\right\|,\left\|\left(B_{2}\right)\right\|\right\}$, with $\|\cdot\|$ denoting the infinity norm since $\mu$ is bounded from below by the spectral radii and from above by the infinity norm of the metrics $B_{0}, B_{1}$, and $B_{2}$. So, $\max \left\{\rho\left(B_{0}\right), \rho\left(B_{1}\right), \rho\left(B_{2}\right)\right\}=\max (|(3 / 4)+9 w|,|(3 / 2)-18 w|)$ and $\max \left(\left\|B_{0}\right\|,\left\|B_{1}\right\|,\left\|B_{2}\right\|\right)=\max (|(3 / 2)-18 w|,|(3 / 4)+9 w|)$. Then, by [20], we have $\mu=\max (|(3 / 2)-18 w|,|(3 / 4)+9 w|)$. So, Hölder regularity of the scheme $S_{m}$ is computed by $r=2 m+1-\log _{3}(\mu)$, where $\mu$ is defined as

$$
\begin{cases}\mu=\frac{3}{2}-18 w & \text { if }-\frac{3}{36}<w \leq \frac{1}{36} \\ \mu=\frac{3}{4}+9 w & \text { if } \frac{1}{36}<w<\frac{9}{36}\end{cases}
$$

which completes the proof.

Corollary 1. The family of schemes $S_{m}$ corresponding to (1) is $C^{2 m}$ continuous if and only if $1 \leq \mu<3$, i.e., if and only if $-(3 / 36)<w<(9 / 36)$.

Theorem 4. The limit stencils providing the evaluations of the basic limit function of the 3-point scheme (5) at integers and half integers are

$$
\begin{aligned}
& \left\{\left(\frac{3 w}{8}+\frac{5}{32}\right),\left(\frac{11}{16}-\frac{3 w}{4}\right),\left(\frac{3 w}{8}+\frac{5}{32}\right)\right\} \\
& \left\{\left(\frac{3 w}{16}+\frac{1}{64}\right),\left(\frac{31}{64}-\frac{3 w}{16}\right),\left(\frac{31}{64}-\frac{3 w}{16}\right),\left(\frac{3 w}{16}+\frac{1}{64}\right)\right\}
\end{aligned}
$$

respectively.

Proof. The local subdivision matrices for limit stencils of 3point scheme (5) at integers and half integers are $f_{I}^{k+1}=$ $S_{I} f_{I}^{k}$ and $f_{I / 2}^{k+1}=S_{I / 2} f_{I / 2}^{k}$, respectively, where

$$
\begin{aligned}
& S_{I}=\left[\begin{array}{lll}
\frac{w}{3}+\frac{13}{36} & \frac{11}{18}-\frac{2 w}{3} & \frac{w}{3}+\frac{1}{36} \\
\frac{w}{3}+\frac{5}{36} & \frac{13}{18}-\frac{2 w}{3} & \frac{w}{3}+\frac{5}{36} \\
\frac{w}{3}+\frac{1}{36} & \frac{11}{18}-\frac{2 w}{3} & \frac{w}{3}+\frac{13}{36}
\end{array}\right], \\
& f_{I}^{k+1}=\left[\begin{array}{c}
f_{-1}^{k+1} \\
f_{0}^{k+1} \\
f_{1}^{k+1}
\end{array}\right], \\
& f_{I}^{k}=\left[\begin{array}{c}
f_{-1}^{k} \\
f_{0}^{k} \\
f_{1}^{k}
\end{array}\right] \text {, }
\end{aligned}
$$




$$
\begin{aligned}
S_{I / 2} & =\left[\begin{array}{cccc}
\frac{w}{3}+\frac{5}{36} & \frac{13}{18}-\frac{2 w}{3} & \frac{w}{3}+\frac{5}{36} & 0 \\
\frac{w}{3}+\frac{1}{36} & \frac{11}{18}-\frac{2 w}{3} & \frac{w}{3}+\frac{13}{36} & 0 \\
0 & \frac{w}{3}+\frac{13}{36} & \frac{11}{18}-\frac{2 w}{3} & \frac{w}{3}+\frac{1}{36} \\
0 & \frac{w}{3}+\frac{5}{36} & \frac{13}{18}-\frac{2 w}{3} & \frac{w}{3}+\frac{5}{36}
\end{array}\right], \\
f_{I / 2}^{k+1}= & {\left[\begin{array}{c}
f_{-3 / 2}^{k+1} \\
f_{-1 / 2}^{k+1} \\
f_{1 / 2}^{k+1} \\
f_{3 / 2}^{k+1}
\end{array}\right], } \\
f_{I / 2}^{k}= & {\left[\begin{array}{c}
f_{-3 / 2}^{k} \\
f_{-1 / 2}^{k} \\
f_{1 / 2}^{k} \\
f_{3 / 2}^{k}
\end{array}\right] . }
\end{aligned}
$$

The eigenvalues of the matrices $S_{I}$ and $S_{I / 2}$ are $\{1,(1 / 9),(1 / 3)\}$ and $\{(1 / 3), 1,(1 / 9),-(2 w / 3)+(1 / 18)\}$, respectively. The eigenvectors of local subdivision matrices $S_{I}$ and $S_{I / 2}$ corresponding to eigenvalues are

$$
\begin{aligned}
Q_{I} & =\left[\begin{array}{ccc}
1 & 1 & -1 \\
1 & \frac{12 w+5}{12 w-11} & 0 \\
1 & 1 & 1
\end{array}\right], \\
Q_{I / 2} & =\left[\begin{array}{cccc}
-1 & 1 & 1 & -1 \\
-\frac{1}{3} & 1 & \frac{12 w+1}{12 w-31} & -\frac{12 w+1}{12 w-7} \\
\frac{1}{3} & 1 & \frac{12 w+1}{12 w-31} & \frac{12 w+1}{12 w-7} \\
1 & 1 & 1 & 1
\end{array}\right] .
\end{aligned}
$$

The inverse of $Q_{I}$ and $Q_{I / 2}$ are

$$
Q_{I}^{-1}=\left[\begin{array}{ccc}
\frac{3 w}{8}+\frac{5}{32} & \frac{11}{16}-\frac{3 w}{4} & \frac{3 w}{8}+\frac{5}{32} \\
\frac{11}{32}-\frac{3 w}{8} & -\frac{11}{16}+\frac{3 w}{4} & \frac{11}{32}-\frac{3 w}{8} \\
-\frac{1}{2} & 0 & \frac{1}{2}
\end{array}\right],
$$

$$
Q_{I / 2}^{-1}=\left[\begin{array}{cccc}
-\frac{3(12 w+1)}{4(12 w+5)} & \frac{3(12 w-7)}{4(12 w+5)} & -\frac{3(12 w-7)}{4(12 w+5)} & \frac{3(12 w+1)}{4(12 w+5)} \\
\frac{3 w}{16}+\frac{1}{64} & \frac{31}{64}-\frac{3 w}{16} & \frac{31}{64}-\frac{3 w}{16} & \frac{3 w}{16}+\frac{1}{64} \\
\frac{31}{64}-\frac{3 w}{16} & -\frac{31}{64}+\frac{3 w}{16} & -\frac{31}{64}+\frac{3 w}{16} & \frac{31}{64}-\frac{3 w}{16} \\
\frac{12 w-7}{4(12 w+5)} & -\frac{3(12 w-7)}{4(12 w+5)} & \frac{3(12 w-7)}{4(12 w+5)} & -\frac{12 w-7}{4(12 w+5)}
\end{array}\right]
$$

For the decomposition of matrices $S_{I}$ and $S_{I / 2}$, we need $\Delta_{I}$ and $\Delta_{I / 2}$, respectively, where $\Delta_{I}$ and $\Delta_{I / 2}$ are the scalar matrices in which eigenvalues are arranged diagonally. Therefore, now compute $\lim _{k \longrightarrow \infty} \Delta_{I}^{k}$ and $\lim _{k \longrightarrow \infty} \Delta_{I / 2}^{k}$ :

$$
\Delta_{I}^{k}=\left[\begin{array}{ccc}
1 & 0 & 0 \\
0 & \left(\frac{1}{9}\right)^{k} & 0 \\
0 & 0 & \left(\frac{1}{3}\right)^{k}
\end{array}\right],
$$

$$
\Delta_{I / 2}^{k}=\left[\begin{array}{cccc}
\left(\frac{1}{3}\right)^{k} & 0 & 0 & 0 \\
0 & 1 & 0 & 0 \\
0 & 0 & \left(\frac{1}{9}\right)^{k} & 0 \\
0 & 0 & 0 & \left(-\frac{2 w}{3}+\frac{1}{18}\right)^{k}
\end{array}\right] \text {. }
$$

This implies

$$
\begin{aligned}
\lim _{K \rightarrow \infty} \Delta_{I}^{k} & =\left[\begin{array}{lll}
1 & 0 & 0 \\
0 & 0 & 0 \\
0 & 0 & 0
\end{array}\right], \\
\lim _{K \rightarrow \infty} \Delta_{I / 2}^{k} & =\left[\begin{array}{llll}
0 & 0 & 0 & 0 \\
0 & 1 & 0 & 0 \\
0 & 0 & 0 & 0 \\
0 & 0 & 0 & 0
\end{array}\right] .
\end{aligned}
$$

Since $f_{I}^{k+1}=S_{I} f_{I}^{k}$, therefore, $f_{I}^{k+1}=S_{I}^{k} f_{I}^{0}$. This implies 


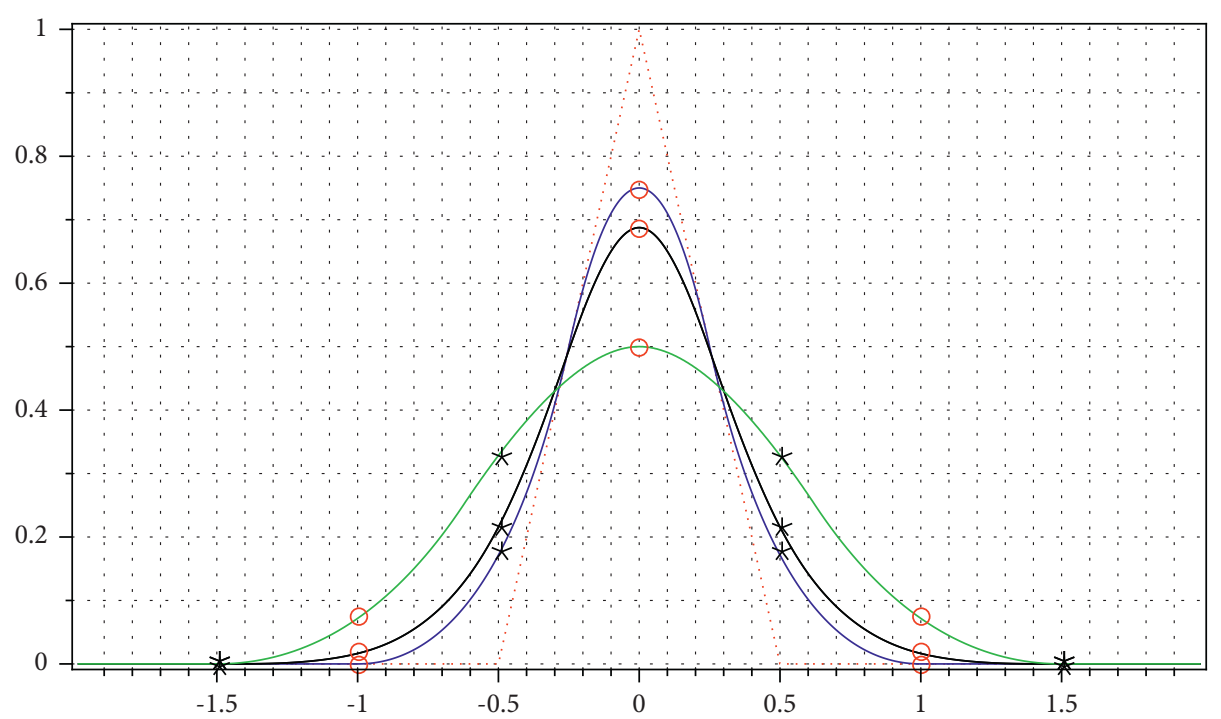

Figure 2: Basic limit functions produced by the 3-point scheme corresponding to $S_{1}$ for different values of parameter $w$. The red line denotes the initial polyline and the blue, black, and green lines represent the basic limit function produced with $w=-(1 / 12), 0,(3 / 12)$, respectively. The circles denote the evaluations of the basic limit function at integers; the asterisks denote the evaluations of the basic limit function at half integers.

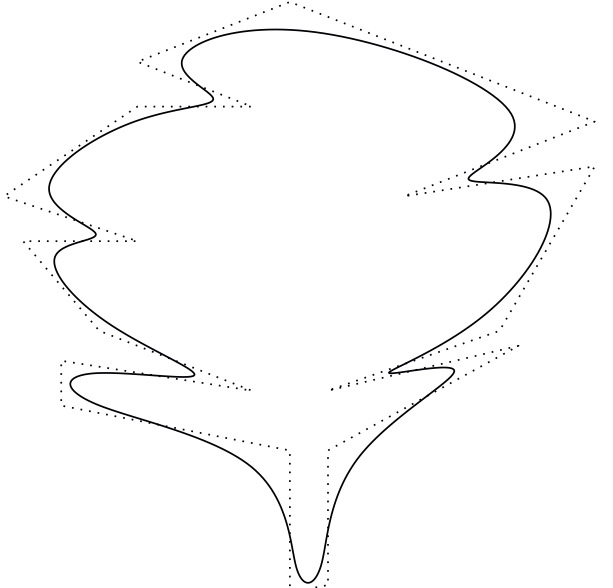

(a)

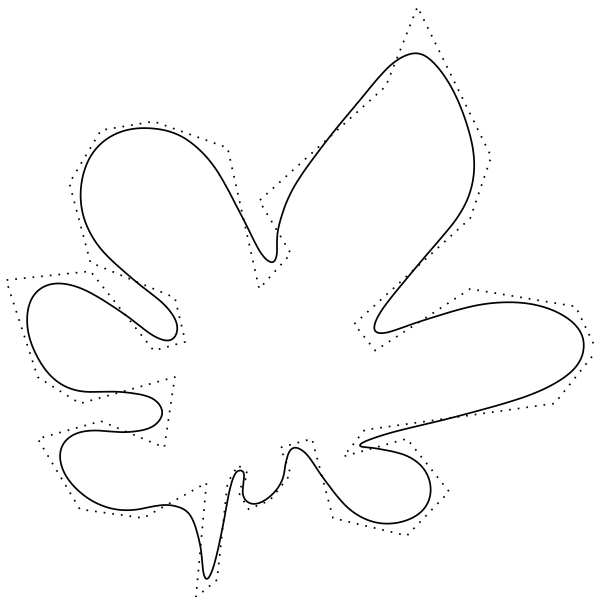

(c)

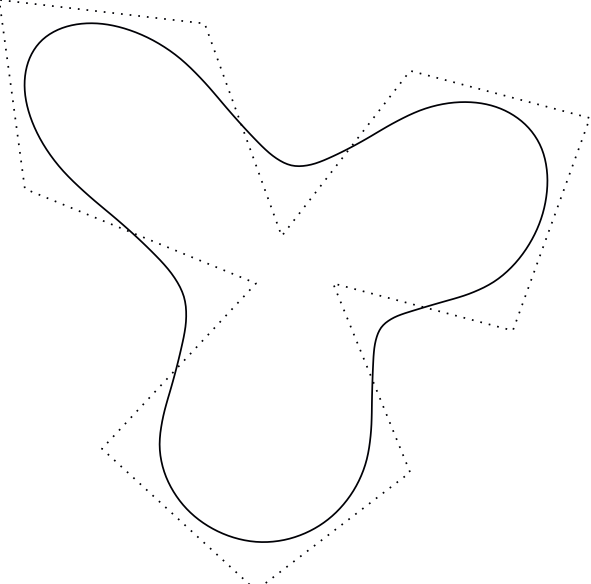

(b)

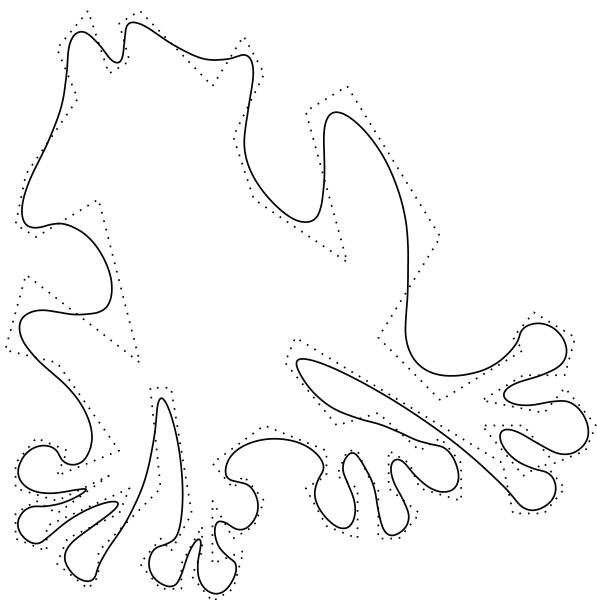

(d)

Figure 3: (a-d) Limit curves for close polygons produced by the scheme corresponding to $S_{1}$. 


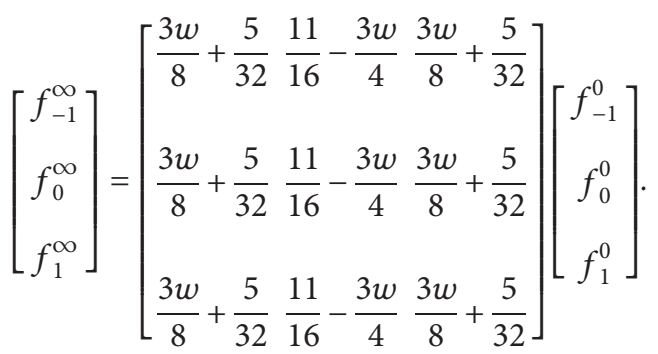

Similarly, $f_{I / 2}^{k+1}=S_{I / 2} f_{I / 2}^{k}$. Therefore, $f_{I / 2}^{k+1}=S_{I / 2}^{k} f_{I / 2}^{0}$. This implies

$$
\left[\begin{array}{l}
f_{-(3 / 2)}^{\infty} \\
f_{-(1 / 2)}^{\infty} \\
f_{(1 / 2)}^{\infty} \\
f_{(3 / 2)}^{\infty}
\end{array}\right]=\left[\begin{array}{lllll}
\frac{3 w}{16}+\frac{1}{64} & \frac{31}{64}-\frac{3 w}{16} & \frac{31}{64}-\frac{3 w}{16} & \frac{3 w}{16}+\frac{1}{64} \\
\frac{3 w}{16}+\frac{1}{64} & \frac{31}{64}-\frac{3 w}{16} & \frac{31}{64}-\frac{3 w}{16} & \frac{3 w}{16}+\frac{1}{64} \\
\frac{3 w}{16}+\frac{1}{64} & \frac{31}{64}-\frac{3 w}{16} & \frac{31}{64}-\frac{3 w}{16} & \frac{3 w}{16}+\frac{1}{64} \\
\frac{3 w}{16}+\frac{1}{64} & \frac{31}{64}-\frac{3 w}{16} & \frac{31}{64}-\frac{3 w}{16} & s \frac{3 w}{16}+\frac{1}{64}
\end{array}\right]\left[\begin{array}{l}
f_{-(3 / 2)}^{0} \\
f_{-(1 / 2)}^{0} \\
f_{(1 / 2)}^{0} \\
f_{(3 / 2)}^{0}
\end{array}\right] .
$$

Hence, the limit stencils providing the evaluations of the basic limit function of the 3-point scheme (5) at integers and half integers are $\{((3 w / 8)+(5 / 32)),((11 / 16)-(3 w / 4))$, $((3 w / 8)+(5 / 32))\}$ and $\{((3 w / 16)+(1 / 64)),((31 / 64)-(3 w /$ $16)),((31 / 64)-(3 w / 16)),((3 w / 16)+(1 / 64))\}$, respectively, which completes the proof.

In Figure 2, we present the basic limit function of the proposed 3-point ternary approximating subdivision scheme for different values of $w$ and show its evaluations at integers and half integers which coincide with the limit stencils computed in Theorem 4.

\section{Comparison with Existing Schemes}

Here, we will present the comparison of our proposed family of ternary subdivision schemes with existing ternary subdivision schemes in the form of theoretical properties and visual performances. In Table 1, we present comparison of same complexity subdivision schemes. From Table 1, we see that our proposed family of ternary subdivision schemes has higher continuity, degree of generation, and degree of reproduction compared with the existing subdivision schemes.

In Figure 3, we present some visual performances of proposed 3-point ternary approximating subdivision scheme. In Figure 1, we present the comparison of the proposed 3-point scheme $S_{1}$ with 3-point scheme $G_{3}$ presented in [5] and 3-point scheme $S_{0}^{3}$ presented in [17]. Here, black dotted lines show the initial polygon, red solid lines are the limit curve of 3-point scheme $S_{0}^{3}$ presented in [17], and blue solid lines are the limit curve of 3-point scheme $G_{3}$ presented in [5]. Mathematically, the continuity of all 3-point schemes is the same, which is $C^{2}$ continuity.

\section{Conclusions}

In this paper, we presented a general formula for derivation of parametric family of ternary subdivision schemes. We presented the complete analysis of the proposed family of parametric ternary subdivision schemes. We also presented the comparison with exiting ternary subdivision schemes. Comparison shows that our proposed family has high continuity, degree of generation and reproduction compared with the same type exiting subdivision schemes. From Table 1, we observed that our proposed 3-point and 5-point schemes have $C^{2}$ and $C^{4}$ continuity, respectively, which are similar to the schemes presented in $[1,5,9,10,17,18]$. Our 6-point scheme has $C^{6}$ continuity which is greater than the 6-point schemes presented in $[4,9]$. Similarly, the generation degree of our proposed schemes is also higher than the exiting schemes.

\section{Data Availability}

The data used to support the findings of the study are included within this paper.

\section{Conflicts of Interest}

The authors declare that they have no conflicts of interest regarding the publication of this paper.

\section{References}

[1] M. F. Hassan, I. P. Ivrissimitzis, N. A. Dodgson, and M. A. Sabin, "An interpolating 4-point $C^{2}$ ternary sationary subdivision scheme," Computer Aided Geometric Design, vol. 19, no. 1, pp. 1-18, 2002.

[2] H. Wang and K. Qin, "Improved ternary subdivision interpolation scheme," Tsinghua Science and Technology, vol. 10, no. 1, pp. 128-132, 2005.

[3] K. P. Ko, B.-G. Lee, and G. J. Yoon, “A ternary 4-point approximating subdivision scheme," Applied Mathematics and Computation, vol. 190, no. 2, pp. 1563-1573, 2007.

[4] G. Mustafa and P. Ashraf, "A new 6-point ternary interpolating subdivision scheme and its differentiability," Journal of Information and Computing Science, vol. 5, pp. 199-210, 2010.

[5] G. Mustafa, A. Ghaffar, and F. Khan, "The odd-point ternary approximating schemes," American Journal of Computational Mathematics, vol. 1, no. 2, pp. 111-118, 2011.

[6] J. Pan, S. Lin, and X. Luo, "A combined approximating and interpolating subdivision scheme with C2 continuity $C^{2}$ continuity," Applied Mathematics Letters, vol. 25, no. 12, pp. 2140-2146, 2012.

[7] S. S. Siddiqi, U. Idrees, and K. Rehan, "Generation of fractal curves and surfaces using ternary 4-point interpolatory subdivision scheme," Applied Mathematics and Computation, vol. 246, pp. 210-220, 2014.

[8] S. S. Siddiqi, S. Siddiqui, and N. Ahmad, "Fractal generation using ternary 5-point interpolatory subdivision scheme," Applied Mathematics and Computation, vol. 234, pp. 402-411, 2014.

[9] S. Siddiqi and M. Younis, "Construction of ternary approximating subdivision schemes," UPB Scientific Bulletin, Series A, vol. 76, no. 1, pp. 1223-7027, 2014. 
[10] K. Rehan and S. Siddiqi, "A family of ternary subdivision schemes for curves," Applied Mathematics and Computation, vol. 270, pp. 14-123, 2015.

[11] P. Ashraf, B. Nawaz, D. Baleanu et al., "Analysis of geometric properties of ternary four-point rational interpolating subdivision scheme," Mathematics, vol. 8, pp. 1-19, 2020.

[12] P. Ashraf, M. Sabir, A. Ghaffar, K. S. Nisar, and I. Khan, "Shape-preservation of the four-point ternary interpolating non-stationary subdivision scheme," Frontiers in Physics, vol. 202010 pages, 2020.

[13] J. Tan and G. Tong, "A nonstationary ternary 4-point shapepreserving subdivision scheme," Journal of Mathematics, vol. 2021, Article ID 6694241, 10 pages, 2021.

[14] H. M. Tariq, R. Hameed, and G. Mustafa, "A new paradigm to design a class of combined ternary subdivision schemes," Journal of Mathematics, vol. 2021, Article ID 6679201, 19 pages, 2021.

[15] R. Hameed, G. Mustafa, J. Deng, and S. Ali, "Recursive process for constructing the refinement rules of new combined subdivision schemes and its extended form," Journal of Mathematics, vol. 2021, Article ID 6639706, 23 pages, 2021.

[16] G. Mustafa, S. T. Ejaz, S. Kouser, S. Ali, and M. Aslam, "Subdivision collocation method for one-dimensional Bratu's problem," Journal of Mathematics, vol. 2021, Article ID 3497017, 8 pages, 2021.

[17] M. Asghar and G. Mustafa, "Family of $a$-ary univariate subdivision schemes generated by Laurent polynomial," Mathematical Problems in Engineering, vol. 2018, Article ID 7824279, 11 pages, 2018.

[18] M. F. Hassan and N. A. Dodgson, "Ternary and three-point univariate subdivision schemes," in Curve and Surface Fitting: Sant-Malo 2002, A. Cohen, J. -L. Merrien, and L. L. Schumaker, Eds., pp. 199-208, Nashboro Press, Brentwood, TN, USA, 2003.

[19] N. Dyn and D. Levin, "Subdivision schemes in geometric modelling," Acta Numerica 2002, vol. 11, pp. 73-144, 2002.

[20] O. Rioul, "Simple regularity criteria for subdivision schemes," SIAM Journal on Mathematical Analysis, vol. 23, no. 6, pp. 1544-1576, 1992. 